\title{
Driving Risk Assessment in Work Zones Using Cloud Model
}

\author{
Chi Zhang $\mathbb{D}^{1},{ }^{1}$ Hong Zhang, ${ }^{1}$ Xiongying Ma, ${ }^{1}$ Min Zhang, ${ }^{2}$ and Shiwei Wang $\mathbb{D}^{1}$ \\ ${ }^{1}$ Key Laboratory for Special Area Highway Engineering of Ministry of Education, Chang'an University, Xian 710064, China \\ ${ }^{2}$ Traffic Engineering Research Institute, School of Highway, Chang'an University, 710064, China \\ Correspondence should be addressed to Chi Zhang; zhangchi@chd.edu.cn
}

Received 20 December 2017; Revised 29 May 2018; Accepted 12 July 2018; Published 16 August 2018

Academic Editor: Gabriel Luque

Copyright (C) 2018 Chi Zhang et al. This is an open access article distributed under the Creative Commons Attribution License, which permits unrestricted use, distribution, and reproduction in any medium, provided the original work is properly cited.

\begin{abstract}
Work zones are prone to traffic accidents. They are considered as dangerous parts of expressways not only for drivers but also for highway construction workers, as they face a higher risk of traffic accidents in work zones. In order to identify the driving risks and to provide guidance to detect traffic risks in work zone, a comprehensive risk assessment method based on cloud model is developed to examine the driving risks in work zones. The proposed model relies on three parameters to determine the driving risks in work zones, namely, coefficient of variation of speed, deceleration, and minimum safety distance. VISSIM simulation software is used as a tool to construct the work zone driving conditions and the reverse cloud model is used to divide the concept and concept jump. The maximum activation intensity is considered as the base factor to determine the core risk level. Other activation intensities are used as a basis to optimize the edge level effect and generate a comprehensive function. The reconstruction area in Anhui Province is used as a case study to assess the driving risks in three expressway work zones. The results revealed that the risk scores of the three work zones 1, 2, and 3 are 48.48, 62.49, and 34.33, respectively. The results obtained by the developed driving risk assessment model are in good agreement with the experimental results. Hence, the model proposed in this paper can accurately assess the driving risks in work zones using a more scientific and intuitive approach, which provides an excellent tool to design safe expressway work zones.
\end{abstract}

\section{Introduction}

The recent rapid development of expressway engineering in China has led to significant increase in the total distance traveled on national expressways that reached 4.4639 million $\mathrm{km}$ by the end of 2015, with an increase of 107.7 thousand $\mathrm{km}$ compared to 2014. Road reconstruction projects are increasing annually despite the stable growth in new expressway projects. Work zones are particular traffic management areas designated for expressway reconstruction and expansion projects. As road reconstruction and expansion projects do not usually interrupt traffic, the impact of such projects on driving is relatively significant. Hence, work zones are considered as dangerous parts of expressways not only for drivers but also for highway construction workers as they face a higher risk of traffic accidents in work zones. Therefore, assessing driving risks is necessary to improve work zones safety in expressways expansion and construction projects.

Most of the current research conducted on work zone driving risks is based on driving risk analysis method for normal sections of expressways. On one hand, the macro traffic risk analysis method relies primarily on the significance and discrepancy of vehicle speed, cross-sectional vehicle speed, and running speed to assess work zone risks. Specific indicators include standard deviation (SD), coefficient of variation $(\mathrm{CV})$, and operating speed. On the other hand, the micro risk analysis mainly uses traffic conflict technology to explore the dangerous spots. The performance indicators for this method include time headway (THW) and time to collision (TTC), maximum deceleration rate of collision presented by the deceleration rate to avoid crash (DRAC), and minimum equivalent safety distance presented by the minimum safety distance equation (MSDE).

In 1994, Lamm and Smith applied the risk theory to assess the risks in roads with circular curves, quantified the risk indicators, and finally evaluated the driving risk of circular curve sections [1]. In 2004, Steenbergen et al. used the traffic accident statistics and GIS technology to identify the dangerous areas of urban road network in Belgium [2]. In 2007, Cunto and Saccomanno conducted micro-intersections 
simulation and risk analysis using indicators such as DRAC [3]. In 2009, based on the risk theory, Ismail set the sight distance index under different vertical curves to assess the road traffic risk [4]. In 2012, Effati et al. considered five road alignment elements and three environmental factors and proposed the corresponding evaluation criteria using GIS and fuzzy reasoning methods to identify dangerous sections [5]. In 2013, Agarwal et al. divided the road into line and curve sections and cross sections to determine the effective factors and used the analytic hierarchy process (AHP) to determine the influence of each factor, then established the assessment conditions for the safety factors, and finally used risk coefficient to determine the dangerous sections [6].

Scholars in China have also conducted a lot of valuable research on driving risk assessment. In 2007, Yu et al. statistically analyzed traffic data of two conservation units and established a safety maintenance prediction model based on the vehicle operating characteristics in work zones [7]. In 2013, Hanqing improved the TTC and DARC conflict calculation model and then proposed a traffic conflict model based on TTC [8]. Bing and Peikun established a traffic accident risk prediction model for road work zones based on the grey system theory $[9,10]$. Zhang adopted a five-point method to calibrate the desired work zone speed, which can reflect the character of traffic flow in work zones [11].

The original intelligent discrimination methods (e.g., genetic algorithm, Bayesian algorithm) have great advantages in solving complex problems as information processing and pattern recognition in the road area. However, those methods are mainly employed for identifying and predicting traffic incidents, poor meteorological forecasting, and other aspects of road traffic risk research yet lacking a practically applicable and accurate method for road micro driving risk assessment.

Because of the obvious randomness of traffic flow and driver behavior in work zones, the level of driving risk is ambiguous; thus, the randomness and fuzziness in the road environment must be considered in work zones risk assessment. This paper explores a reasonable approach to classify the driving risk state of work zones. An artificial intelligence cloud model can convert qualitative concepts into quantitative data. The digital characteristics of cloud can fully reflect the fuzziness and randomness of work zones traffic. Furthermore, the cloud generator realizes the indefinite membership degree representation, which can be applied to classify the driving risks in work zones.

In their original literature about cloud theory, Deyi et al. introduced the digital characteristics of cloud concept, digital cloud-features, and cloud generator implementation process, in addition to developing and analyzing the cloud generator using mathematical mode [12]. As the cloud theory developed, the application of cloud model has been extended by many scholars. In recent years, the cloud model has been successfully applied in the fields of intelligent control and prediction, data mining, system evaluation, etc.

In 2009, Xiaodong considered the highway alignment condition and horizon as the index and built a prediction mode of operating speed based on cloud model [13]. In 2012, Jinchang offered a comparison between the fuzzy evaluation and fuzzy comprehensive evaluation based on cloud model and then provided an example to verify the advantages of cloud model in the fuzzy comprehensive assessment [14]. In 2013, Shibin applied the fuzziness, randomness, and uncertainty characteristics of cloud model to the trust mechanism and realized the quantitative and qualitative transformation of trust [15]. In 2014, Qiuwen used the multilevel fuzzy comprehensive evaluation method of cloud model to perform reservoir-induced earthquake analysis of each unit of the Three Gorges Reservoir area [16]. In 2014, Xingli combined the principal component analysis and the expert investigation method to improve the AHP and proposed a method to assess the earthquake damage to expressway based on the cloud model and used the cloud model to complete the earthquake damage assessment in the seismic area [17]. In 2014, Zhengjie conducted risk assessment for a railway signal system and employed the cloud model to perform risk assessment using fuzzy comprehensive evaluation method [18]. In 2015, Yuan applied the cloud model to the mountain railway route selection and adopted projection method to optimize the railway route scheme, in addition to developing an evaluation model for mountain railway route selection by synthetically considering the quantitative and qualitative indexes [19]. In 2018, Gong proposed a new simple cloud model called triangular cloud model and the forward triangular cloud transformation algorithm is presented. Then the author used two simulation examples to state the feasibility of the triangular cloud [20].

A lot of research has been conducted on the application of cloud model in different fields; however, the model application to driving risk assessment in work zones has not been investigated yet. Therefore, this study adopts the cloud model to identify work zone driving risks. This approach allows avoiding the problems associated with the uncertainty analysis and combines the fuzzy, random, and discrete characters of work zone driving risks. Based on measured data, the Gauss cloud transform method is employed to analyze the data and complete the driving risk assessment in this study using the cloud model.

\section{Cloud Model for Driving Risk Assessment}

2.1. Cloud Model Theory. Cloud model is an uncertainty transformation model between qualitative concepts and their quantitative expressions.

Let $\mathrm{U}_{1}$ be a quantitative domain represented by an exact number, and let $\mathrm{C}$ be a qualitative concept on $\mathrm{U}_{1}$. If the values of quantitative $\mathrm{x} \in \mathrm{U}_{1}$ and $\mathrm{X}$ are a random implementation of the qualitative concept $\mathrm{C}$, certainty $\mu(\mathrm{x}) \in[0,1]$ of $\mathrm{x}$ in $\mathrm{C}$ has a stable tendency of random numbers. The distribution of $\mathrm{X}$ in field $U_{1}$ is called the cloud. Each $\mathrm{x}$ is called a cloud droplet [21].

$$
\mu: U_{1} \longrightarrow[0,1] \quad \forall x \in U x \longrightarrow \mu(x)
$$

The cloud is characterized by 3 numerical characteristics: expected Ex, entropy En, and super entropy He. Ex is the expectation of cloud droplet in the theory of domain space; En is uncertainty measure of qualitative concepts determined 


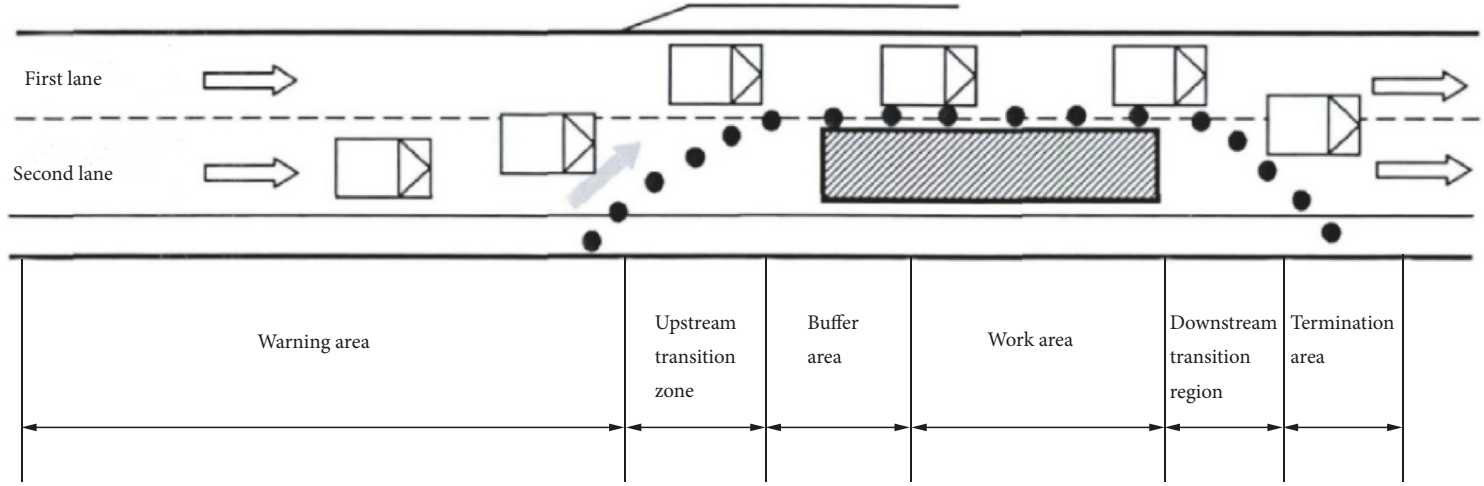

FIGURE 1: Layout of work zone.

by the randomness and fuzziness of the concept. He is uncertainty measure of entropy. The transformation process from qualitative concept to quantitative representation is called forward cloud generator, which is the digital characteristic of cloud droplets produced by cloud. The transformation process from quantitative representation to the qualitative concept is called backward cloud generator, which calculates digital characteristics of cloud from the cloud droplets.

Another important numerical characteristic of cloud model is degree of ambiguity CD. Entropy and hyper entropy are the uncertainty measures of concepts. The ratio of hyper entropy to entropy reflects the maturity of concepts.

When the cloud model is characterized, $\mathrm{He}=\mathrm{En} / 3$ is a characteristic value with distinct demarcation properties. Therefore, the degree of ambiguity $\mathrm{CD}$ is proposed to measure the degree of discretization of the concept epitaxy. Equation (2) is calculated.

$$
C D=\frac{3 H e}{E n}
$$

(1) When $\mathrm{He}=0$, the cloud droplet presents a strict Gauss distribution.

(2) When $0<\mathrm{He}<\mathrm{En} / 3$, the certainty of data to the concept existing uncertainty shows a pan Gauss distribution.

(3) When $\mathrm{He}=\mathrm{En} / 3$, the cloud droplets are gradually atomized, and the conceptual maturity is relatively low.

(4) When $\mathrm{He}>\mathrm{En} / 3$, the concept is completely misty and cannot be regarded as a mature concept.

When $\mathrm{CD}=0$, the concept convergence is very mature; when $\mathrm{CD}=1$, the concept extension is dispersed and gradually atomized, and the concept acceptance is low.

Cloud transformation involves decomposing the arbitrary functions into the superposition of basic clouds within a certain error area. When the distribution function is known, cloud transformation can be used to generate concepts from data distribution. Cloud transformation is defined as follows.

The frequency distribution function $\mathrm{f}(\mathrm{x})$ of a data attribute $\mathrm{X}$ in the set domain is known according to the distribution of frequency of $\mathrm{X}$, which automatically generates the superposition of a number of clouds $\mathrm{C}\left(E x_{i}, E n_{i}\right.$ and $\left.H e_{i}\right)$ with different granularity. Each cloud represents a concept, which is mathematically expressed by (3), as follows:

$$
f(x) \longrightarrow \sum_{i=1}^{n}\left(a_{i} \times C\left(E x_{i}, E n_{i}, H e_{i}\right)\right)
$$

where $\mathrm{C}$ denotes the set of generating concepts; $\mathrm{AI}$ is the amplitude coefficient; $\mathrm{n}$ is the number of discrete concepts generated after transformation.

2.2. Driving Environment Simulation. Traffic risk refers to the probability and severity of accidents in a certain section of a certain road under certain road traffic conditions. The existence of traffic risk indicates greater possibility of accidents, more severe accidents, and higher driving risk on this section of the road; conversely, fewer accidents will be expected on road sections with low driving risk [22].

The driving environment in work zones is complicated, and the complexity of risks associated with vehicle driving is also considered as uncertain data. Through processing the velocity and deceleration data, the cloud transformation and reasoning technologies were used to assess the vehicle operation risk in work zones, which can clearly reflect the randomness and fuzziness of driving risks. In this study, VISSIM simulation software is used as a tool to construct the driving conditions of a work zone with one lane closed. VISSIM simulation software has two follow-up models, namely, Wiedemann 74 and Wiedemann 99 models. While Wiedemann 74 model is primarily used to simulate urban road traffic, Wiedemann 99 model is mainly used for intercity highway traffic simulation. Therefore, Wiedemann 99 model is adopted in this study as it is typically used to simulate traffic in work zones. The layout of the simulated work zone is shown in Figure 1.

Figure 2 shows a flow chart that summarizes the driving risk assessment process. First of all, finish the acquisition of basic data including work zone data and traffic data. Then according to the basic data, simulate the work zone driving environment by using simulation software. Then select the evaluation factors from macro and micro angles. At last use cloud model to finish the driving risk assessment. 


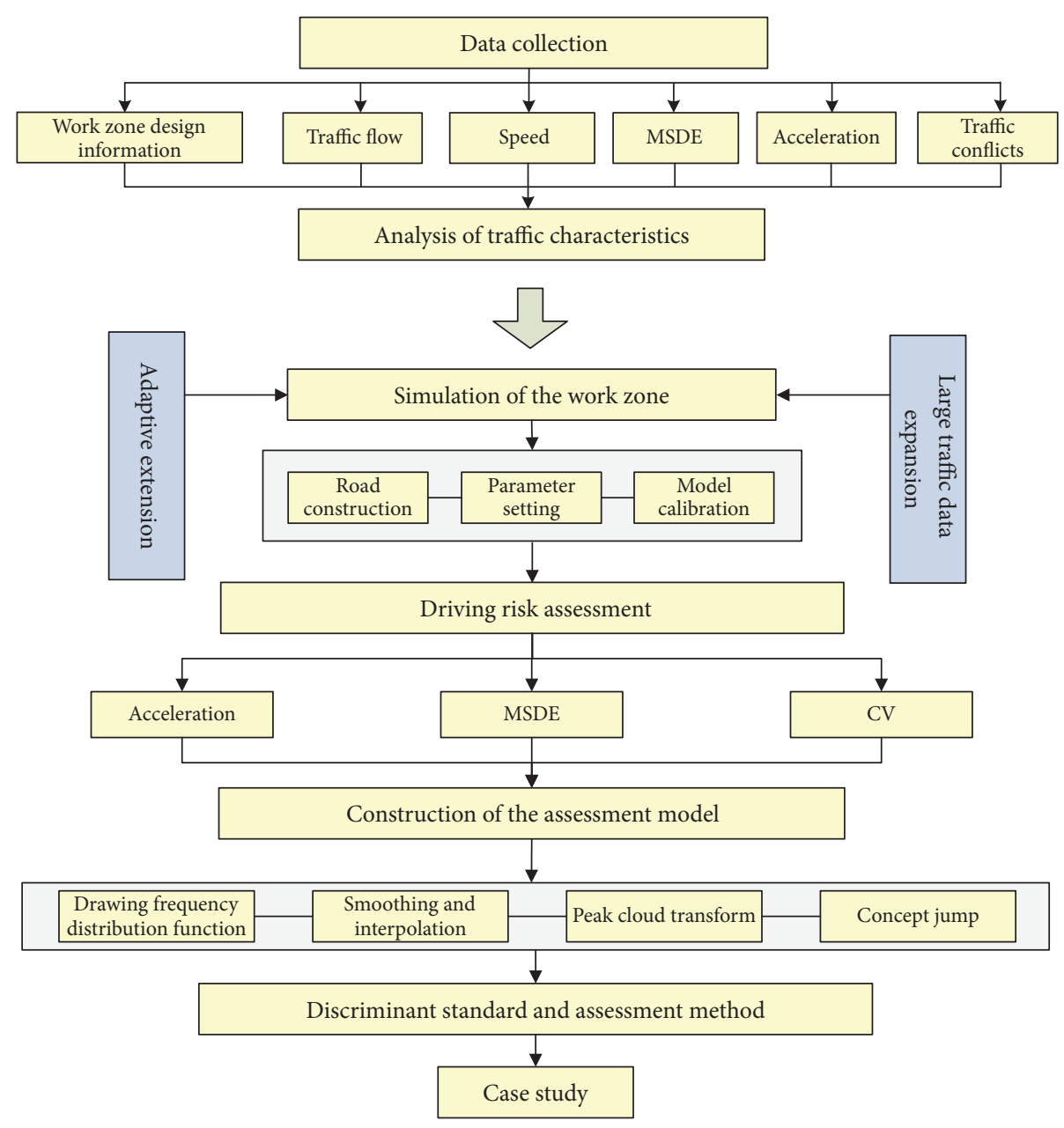

FIGURE 2: Flow chart of driving risk assessment.

The specific process of using the cloud model for driving risk assessment is as follows.

(1) Coefficient of variation is used to analyze the dispersion and continuity of macro traffic flow velocity in work zone, respectively. The deceleration and minimum safety distance equation are used as the basis of the judgment of the risk state of the micro vehicle.

(2) By using the smoothing and interpolating processing of the four discriminant indicator samples, the frequency distribution function of the sample data is obtained, and the peak cloud transformation intelligent algorithm is used to divide the concept of hazard. Then, the four hazard classes are named to jump over the concept. Expected value, entropy, and hyper entropy of cloud model are obtained. Then use the forward cloud generator to establish the construction area traffic hazard identification cloud model.

(3) The maximum activation intensity is the criterion of the core hazard level. The other activation intensity is the basis of the edge level effect and the comprehensive function and the discriminant method.

2.3. Factors Identification and Evaluation. The analysis of forms and causes of accidents in expressway work zones indicates that the driving risk is associated with the change in expressway traffic flow at any time, and researchers typically select various traffic flow parameters to characterize the current traffic flow status.

In general, traffic flow parameters related to driving risk can be divided into two categories. The first category comprises macroscopic traffic flow parameters that characterize the risk of traffic flow disturbance. Such parameters are correlated with traffic accident and conflict data, then regressions and other mathematical means are used to establish the prediction model. The other category comprises microscopic traffic flow parameters that characterize the driving risk of a single vehicle. They are extracted from the theory of vehicle dynamics and car-following theory. The driving risk is typically analyzed using the control indexes of critical speed, critical acceleration, and minimum safety distance.

In this study, the acceleration and equal minimum safety distance (MSDE) are selected as micro traffic flow parameters to assess the driving risk. Meanwhile, the macro traffic flow parameters are considered as the coefficients of variation of speed (CV) to assess the driving risk. The combination of micro and macro parameters will ensure the accuracy and reliability of the identified work zone risks [23]. 




(a)

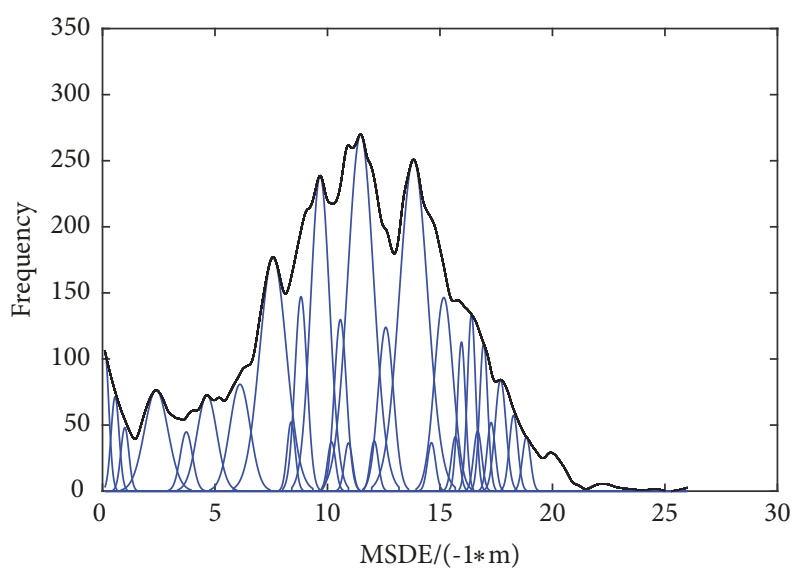

(c)

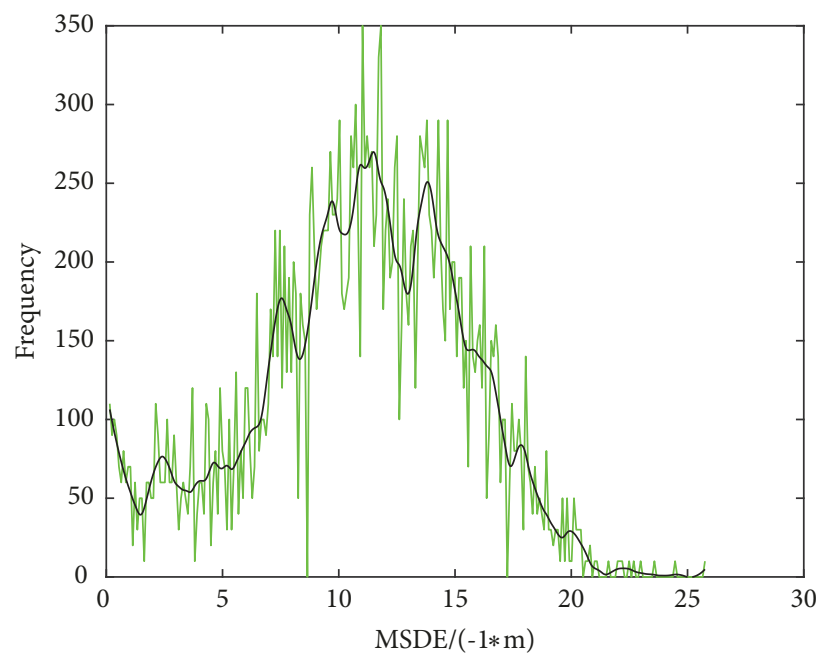

(b)



(d)

FIgure 3: Concept partition process. (a) Frequency distribution histogram of MSDE. (b) Smooth interpolation of MSDE. (c) Cloud transformation of MSDE. (d) Concept jump of MSDE.

2.4. Traffic Conflict. In order to properly identify and assess work zone risks, the vehicle driving state is a critical factor to be considered in assessing driving risks. Traffic conflicts are used as inference condition, as they can directly reflect the dangerous state of vehicle in work zones. Traffic conflicts are mainly divided into two categories, namely, forced confluence conflict and rear-end collision. Forced confluence is the traffic conflict involving lane changing behavior, while rear-ends collision involves the car-following behavior. Traffic conflicts are based on the following static principles: (1) the vehicle must be forced to brake or slow when it is forced to change lane; (2) applying emergency brakes while driving in the same lane to ensure a safe distance from the front vehicle is a conflict.

2.5. Concept Partition. MSDE is selected as an example to explain the concept partition process. First, select the simulation data with "interaction" as BREAKAX corresponding to the MSDE as the original data to draw the MSDE frequency histogram (see Figure 3(a)); then use local polynomial regression and three-spline interpolation algorithm to smooth and interpolate the MSDE data (see Figure 3(b)); next transform the MSDE frequency distribution function into cloud after interpolation (see Figure 3(c)); finally, use the cloud transform mining to obtain the concept number; however, the result does not meet the characteristics of human cognition of concept; thus the concept numbers and conceptual jump must be reduced. Hence, the risk grades are divided into four groups, and the digital characteristics and degree of ambiguity of cloud are extracted (see Figure 3(d)). The concept of MSDE has completed the concept partition. The results of evaluation factors concept partition of CV and acceleration are obtained using this method, as shown in Figures 3 and 4.

The locally weighted regression scatterplot smoothing method is used in this study as it does not produce a clear functional equation, and the data can reflect its own characteristics. It also has some shortcomings as it requires a great deal of data; however, sufficient data can be obtained by the traffic simulation method used in this experiment [24].

Data interpolation methods are near point interpolation, linear interpolation, cubic Hermite interpolation, and cubic 


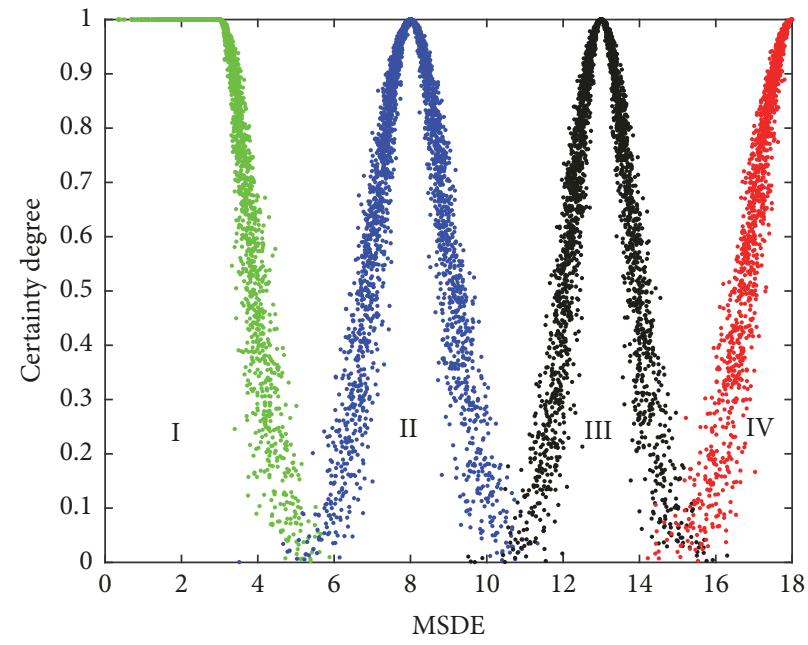

(a)

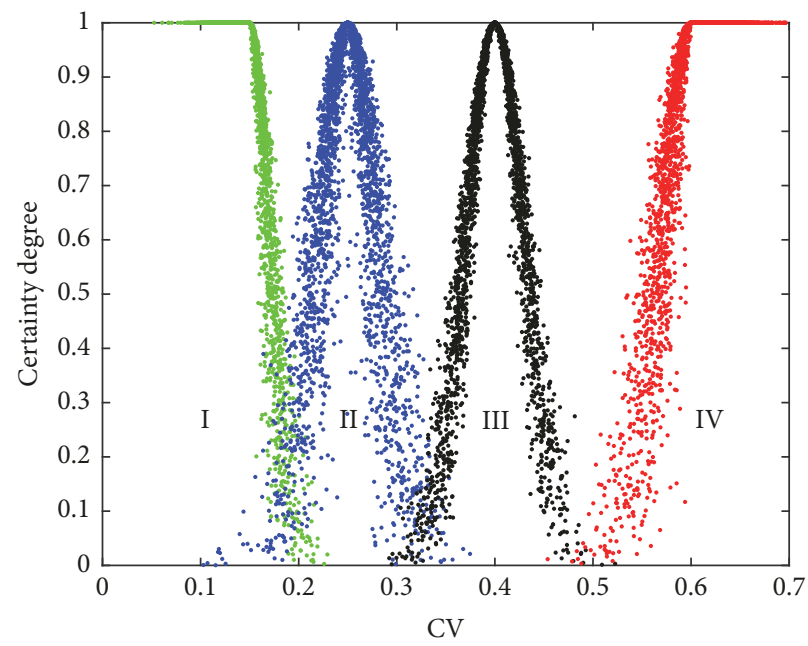

(b)

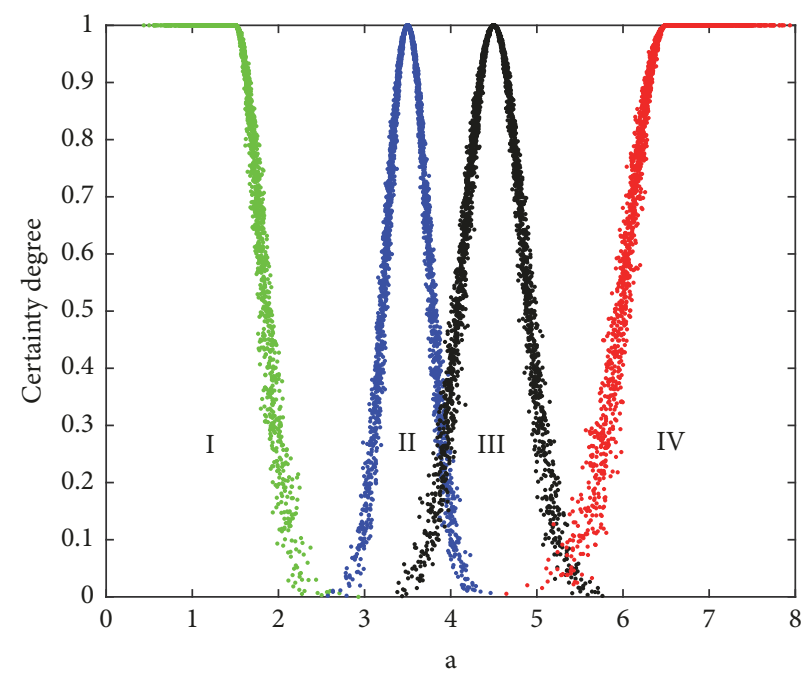

(c)

FIGURE 4: Evaluation cloud of assessment indexes. (a) Evaluation cloud of MSDE. (b) Evaluation cloud of CV. (c) Evaluation cloud of a.

spline interpolation in MATLAB. Both the cubic spline interpolation and derivative value are close to the interpolated function; thus, the method can produce the best approximation effect. Therefore, the cubic spline method is selected in this study. The first-order and second-order derivatives are under continuous condition in order to effectively avoid Runge's phenomenon [25].

The method explained above can be used to obtain the assessment factors concept partition of CV and acceleration. The obtained results are shown in Figure 4 and Table 1.

(1) From the expected value results, the risk level is directly associated with the MSDE numerical range. MSDE is considered less risky if it ranges from 0 to -6 . The analysis and discrimination of coefficient of speed variation and driving risk level are implemented to determine the coefficient of speed variation. Based on the assessment cloud model, partially safe driving conditions with relatively low driving risks are achieved when the coefficient of variation is lower than 0.25 .
(2) From hyper entropy results, the hyper entropy of coefficient of speed variation is the largest in the macro traffic flow parameters. The outer appearance shows that the cloud has the largest thickness with an atomized state. However, the degree of ambiguity of each concept is still to achieve consensus. The hyper entropy of acceleration is the smallest. The concept has higher acceptance.

(3) From the mixed CD results, the concepts of "safe", "less dangerous", and "dangerous" have coefficient of variation of speed of more than 1. It indicates that the concept of extension showed a divergent state, and atomization is more obvious. The concept is formatted in lower acceptance.

2.6. Driving Risk Scores. The maximum activation determination scoring method is adopted to conduct a comprehensive consideration of risk assessment cloud model using three indicators. The scores corresponding to each risk level are listed in Table 2 [26, 27].

The actual assessment index values are entered into each risk discrimination cloud model to obtain the certainty 
TABLE 1: Evaluation factors concept partition.

\begin{tabular}{|c|c|c|c|c|c|}
\hline Assessment factors & Driving risk & Ex & En & $\mathrm{He}$ & $\mathrm{CD}$ \\
\hline \multirow{4}{*}{ a } & safe & 1.5 & 0.30 & 0.040 & 0.40 \\
\hline & Less safe & 3.5 & 0.25 & 0.025 & 0.30 \\
\hline & Less dangerous & 4.5 & 0.35 & 0.035 & 0.30 \\
\hline & dangerous & 6.5 & 0.45 & 0.055 & 0.37 \\
\hline \multirow{4}{*}{ MSDE } & safe & -3 & 0.80 & 0.170 & 0.64 \\
\hline & Less safe & -8 & 0.95 & 0.185 & 0.58 \\
\hline & Less dangerous & -13 & 0.80 & 0.160 & 0.60 \\
\hline & dangerous & -18 & 1.05 & 0.195 & 0.56 \\
\hline \multirow{4}{*}{$\mathrm{CV}$} & safe & 0.15 & 0.02 & 0.005 & 0.75 \\
\hline & Less safe & 0.25 & 0.03 & 0.010 & 1.00 \\
\hline & Less dangerous & 0.40 & 0.03 & 0.005 & 0.50 \\
\hline & dangerous & 0.60 & 0.03 & 0.010 & 1.00 \\
\hline
\end{tabular}

TABLE 2: Driving risk scores.

\begin{tabular}{lcccc}
\hline Driving risk & safe & Less safe & Less dangerous & dangerous \\
\hline Driving risk level & I & II & III & IV \\
Driving risk score & 5 & 10 & 15 & 25 \\
\hline
\end{tabular}

degree and calculate the acceleration index using (4)-(6). The remaining indicators of driving risk score can be calculated by (4) and (5). Finally, (4) is used to calculate the driving risk scores, while the maximum certainty degree is used to determine the risk level. In addition, the sign of (4) [28] is added when the other degree of certainty of activation lies in the ascending cloud of the respective risk level. A negative sign of (6) is used when the other degree of certainty of activity locates the cloud that goes down. The equations used to calculate the driving risk scores are

$$
\begin{aligned}
\mu_{a, j} & =\operatorname{LARGE}\left[\left\{\mu_{a}^{1}, \mu_{a}^{2}, \mu_{a}^{3}, \mu_{a}^{4}\right\}, j\right] \\
R I_{a} & =G_{a, 1}+\sum_{j=2}^{4}\left( \pm G_{a, j} \times \mu_{a, j}\right) \\
R I & =\left(R I_{a}+R I_{M S D E}+R I_{C V}\right) \times \frac{4}{3}
\end{aligned}
$$

\section{Case Study}

The driving risk assessment method is applied to evaluate the work zones in Anhui Province, which is located in Feidong County. The front and back ends for the upstream transition area of three work zones are selected as the data acquisition section. The section contains three work zones with a total length of $10.1 \mathrm{~km}$. A satellite image of this section is shown in Figure 5.

Detailed information about the three construction zones measured in this case study is listed in Table 3.

The vehicle speed, deceleration data, and MSDE data are obtained in this study and then combined with the traffic conflicts detected in the construction area of transition zones. Traffic conflicts are determined based on the statistics of

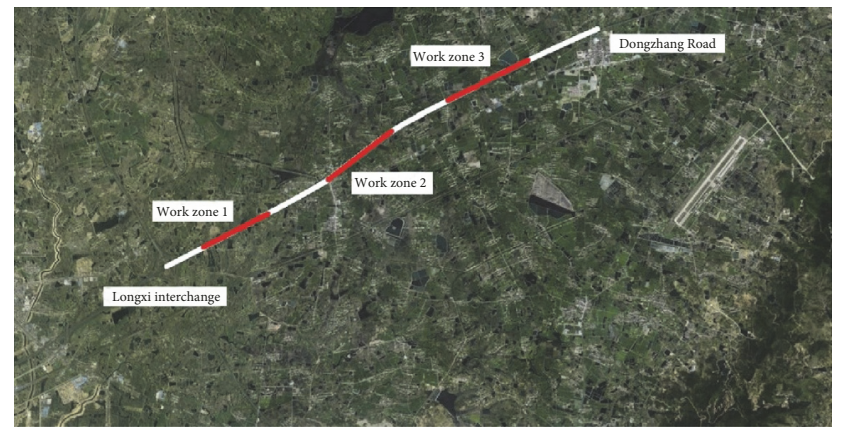

FIGURE 5: Satellite map of the measured road section.

traffic conflict count principles described in the previous section. The coefficient of variation and MSDE were calculated.

The assessment index of each group is entered into the concept to obtain the certainty degree of each index in the cloud model, as listed in Table 4.

(1) The measured data of each index are entered into various risk discrimination cloud models to obtain the degree of certainty information corresponding to each risk assessment level, as listed in Table 4.

(2) Equation (2) is adopted to calculate the maximum value of each index corresponding to the degree of certainty, and the corresponding risk level is considered as the discrimination level.

$$
\begin{aligned}
\mu_{a_{85}, 1} & =\operatorname{LARGE}[\{0,0.469,0.014,0\}, 1] \\
& =0.469 \text { "less safe" } \\
\mu_{M S D E_{85}, 1} & =\operatorname{LARGE}[\{0.412,0.153,0,0\}, 1] \\
& =0.412 \text { "safe" }
\end{aligned}
$$


TABLE 3: Detailed information of measured section.

\begin{tabular}{lccc}
\hline Work zones & length $(\mathrm{km})$ & flow $(\mathrm{veh} / \mathrm{h})$ & Closed mode \\
\hline Work zone 1 & 1.68 & 1624 & Closed half lane \\
Work zone 2 & 1.75 & 1527 & Closed half lane \\
Work zone 3 & 1.72 & 1586 & Closed half lane \\
\hline
\end{tabular}

TABLE 4: Assessment indexes in concept cloud.

\begin{tabular}{|c|c|c|c|c|c|c|c|c|c|c|}
\hline Work zones & $\begin{array}{l}\text { Concept hierarchy } \\
\text { assessing indicator }\end{array}$ & value & I & II & III & IV & Risk level & & $\begin{array}{l}\text { Risk } \\
\text { score }\end{array}$ & $\begin{array}{c}\text { Traffic } \\
\text { conflicts } \\
\end{array}$ \\
\hline \multirow{3}{*}{ Work zone 1} & $a_{85}$ & 3.8 & 0 & 0.461 & 0.232 & 0 & Less safe & 13.48 & \multirow{3}{*}{48.48} & \multirow{3}{*}{42} \\
\hline & $\mathrm{MSDE}_{85}$ & -4.3 & 0.265 & 0.023 & 0 & 0 & safe & 5.23 & & \\
\hline & $\mathrm{CV}$ & 0.479 & 0 & 0 & 0.278 & 0.106 & $\begin{array}{c}\text { Less } \\
\text { dangerous }\end{array}$ & 17.65 & & \\
\hline \multirow{3}{*}{ Work zone 2} & $a_{85}$ & 5.3 & 0 & 0.879 & 0.011 & 0 & $\begin{array}{c}\text { Less } \\
\text { dangerous }\end{array}$ & 15.85 & \multirow{3}{*}{62.49} & \multirow{3}{*}{73} \\
\hline & $\mathrm{MSDE}_{85}$ & -13.6 & 0 & 0 & 0.157 & 0.034 & $\begin{array}{c}\text { Less } \\
\text { dangerous }\end{array}$ & 16.03 & & \\
\hline & $\mathrm{CV}$ & 0.436 & 0 & 0 & 0.653 & 0.041 & $\begin{array}{c}\text { Less } \\
\text { dangerous } \\
\end{array}$ & 14.99 & & \\
\hline \multirow{3}{*}{ Work zone 3} & $a_{85}$ & 4.3 & 0 & 0.001 & 0.685 & 0 & $\begin{array}{c}\text { Less } \\
\text { dangerous }\end{array}$ & 5.32 & \multirow{3}{*}{34.33} & \multirow{3}{*}{31} \\
\hline & $\mathrm{MSDE}_{85}$ & -6.4 & 0 & 0.003 & 0.864 & 0 & safe & 10.56 & & \\
\hline & $\mathrm{CV}$ & 0.302 & 0 & 0.023 & 0.651 & 0 & Less safe & 9.96 & & \\
\hline
\end{tabular}

$$
\begin{aligned}
\mu_{C V, 1} & =L A R G E[\{0,0.032,0.348,0\}, 1] \\
& =0.348 \text { "less dangerous" }
\end{aligned}
$$

(3) The effect of other levels of certainty on the discriminant result is considered, and (3) is used to calculate the risk scores of subindicator.

$$
\begin{aligned}
R I_{a_{85}} & =10+(0 \times 5+0.014 \times 15+0 \times 25) \\
& =10.21 \text { "Rising cloud edge effects" } \\
R I_{M S D E_{85}} & =5+(0.153 \times 5+0 \times 15+0 \times 25) \\
& =6.53 \text { "Rising cloud edge effects" } \\
R I_{C V} & =15+(0 \times 5-0.032 \times 10+0 \times 25) \\
& =14.68 \text { "Falling clouds edge effects" }
\end{aligned}
$$

(4) Equation (4) is used to calculate the risk score.

$$
\begin{aligned}
R I & =\left(R I_{a_{85}}+R I_{M S D E_{85}}+R I_{C V}\right) \times \frac{4}{3} \\
& =(10.21+6.53+14.68) \times \frac{4}{3}=48.48
\end{aligned}
$$

According to the discriminant effect, similar trends are observed between the comprehensive driving risk and measured traffic conflicts. The results indicate that the effect of driving risk assessment is credible, and accidents are more likely to occur in this work zone; thus, drivers should be vigilant to avoid traffic accidents.

\section{Conclusions}

The following conclusions are derived from this study:

(1) Both the macro and micro risk assessment factors have been examined in this study, considering the complexity of traffic conditions and factors influencing traffic safety. The concept jump of three risk assessment factors is completed using the simulation date.

(2) The proposed driving risk assessment method was verified based on three reconstruction work zones included in the case study located in Feidong County of Anhui Province. The driving risk levels and scores can be calculated using the proposed method. The obtained risk scores of the three work zones 1,2 , and 3 were 48.48, 62.49, and 34.33, respectively. Their risk levels agree well with the measured traffic conflicts.

(3) The driving risk assessment model developed in this study can provide a theoretical basis to identify and detect traffic risks in work zones. The accuracy and reliability of work zones risk assessment are improved by the cloud model, which fully considers the randomness, fuzziness, and dispersion of driving risks. It also provides an excellent reference value for further research on safety measures in work zones.

(4) The risk assessment model developed in this study only considers the main factors affecting driving risks in work zones. Therefore, further research is required to address the limitations of this model considering the more assessment factors and weights.

\section{Data Availability}

The evaluation factors concept partition data, the detailed information of measured section data, and the assessment 
index in concept cloud data used to support the findings of this study are included within the article. The code data used to support the findings of this study are available from the corresponding author upon request.

\section{Conflicts of Interest}

The authors declare that there are no conflicts of interest regarding the publication of this paper.

\section{Acknowledgments}

The authors would like to gratefully acknowledge the support provided by Postdoctoral Science Foundation Project (2016M590915), Natural Science Basic Research Program of Shaanxi (S2017-ZRJJ-MS-0603), and Fundamental Research Funds for the Central Universities (310821172002, 310821172201).

\section{References}

[1] R. Lamm and B. L. Smith, "Curvilinear alinement: an important issue for more consistent and safer road characteristic," Transportation Research Record, no. 1445, pp. 12-21, 1994.

[2] T. Steenberghen, T. Dufays, I. Thomas, and B. Flahaut, "Intraurban location and clustering of road accidents using gis: A belgian example," International Journal of Geographical Information Science, vol. 18, no. 2, pp. 169-181, 2004.

[3] F. Cunto and F. F. Saccomanno, "Calibration and validation of simulated vehicle safety performance at signalized intersections," Accident Analysis \& Prevention, vol. 40, no. 3, pp. 11711179, 2008.

[4] K. Ismail and T. Sayed, "Risk-based framework for accommodating uncertainty in highway geometric design," Canadian Journal of Civil Engineering, vol. 36, no. 5, pp. 743-753, 2009.

[5] M. Effati, M. A. Rajabi, F. Samadzadegan, and J. A. Blais, "Developing a Novel Method for Road Hazardous Segment Identification Based on Fuzzy Reasoning and GIS," Journal of Transportation Technologies, vol. 02, no. 01, pp. 32-40, 2012.

[6] P. K. Agarwal, P. K. Patil, and R. Mehar, "A Methodology for Ranking Road Safety Hazardous Locations Using Analytical Hierarchy Process," Procedia - Social and Behavioral Sciences, vol. 104, pp. 1030-1037, 2013.

[7] Y. Ying, M. Wang, J. Mingxin et al., "Study on safety characteristics of expressway maintenance construction area," Chinese Highways, vol. 27, no. 6, pp. 186-189, 2007.

[8] X. Hanqing, Study on Traffic Conflicts and Safety Assessment of Typical Expressway Section, Harbin Institute of Technology, 2013.

[9] W. Biao, X. Hongguo, and D. Tongyan, "Identification of influencing factors of traffic safety in expressway operation area based on DEMATEL-ISM," vol. 10, pp. 130-136, Transportation Systems Engineering and Information, 2010.

[10] W. Biao, X. Hongguo, and D. Tongyan, "Simulation evaluation model of expressway operation risk," Transportation systems engineering and information, vol. 13, no. 3, pp. 151-156, 2013.

[11] C. Zhang, S. Liu, J. Ogle, and M. Zhang, "Micro-simulation of desired speed for temporary work zone with a new calibration method," Promet-Traffic \& Transportation, vol. 28, no. 1, pp. 4961, 2016.
[12] L. Deyi, M. Haijun, and S. Xuemei, "Membership cloud and membership cloud generator," Computer research and development, no. 6, pp. 15-20, 1995.

[13] L. Xiaodong, A Preliminary Study on Cloud Model of Operating Speed Based on Highway Alignment and Sight Distance, Jilin University, 2009.

[14] S. Jinchang, D. Shuxin, and L. Wei, "Fuzzy comprehensive evaluation method and its application based on cloud model," Fuzzy Systems and Mathematics, vol. 26, no. 6, pp. 115-123, 2012.

[15] Z. Shibin and X. Chunxiang, "Research on trust evaluation method based on cloud model," Journal of Computer \& Science, vol. 36, no. 2, pp. 422-431, 2013.

[16] Z. Qiuwen, Z. Yongzhi, and Z. Ming, "Multilevel fuzzy comprehensive evaluation of reservoir induced earthquake risk based on cloud model," Journal of hydraulic engineering, vol. 45, no. 1 , pp. 87-95, 2014.

[17] J. Xingli and X. Jinliang, "Seismic risk assessment of highway in seismic area based on cloud model," Journal of Tongji University Natural Science edition, vol. 42, no. 9, pp. 1352-1358, 2014.

[18] X. Zhengjie, Z. Youpeng, and S. Hongsheng, "Application of fuzzy comprehensive evaluation method in risk assessment based on cloud model," Journal of Safety and Environment, vol. 14, no. 2, pp. 69-72, 2014.

[19] Y. Luo and L.-K. Yao, "Scheme evaluation on railway location design in mountains based on cloud model," Chinese Journal of Engineering Design, vol. 22, no. 4, pp. 372-380, 2015.

[20] Y. Gong, L. Dai, and N. Hu, "A triangular cloud model and cloud generator algorithm," Open Cybernetics and Systemics Journal, vol. 10, pp. 192-201, 2016.

[21] D. Li and Y. Du, Uncertainty artificial intelligence, National Defense Industry Press, 2014.

[22] H. Xiaoqing, L. Benmin, and G. Zhongyin, "Application of traffic simulation in road safety evaluation," Shandong Traffic Science and Technology, vol. 2, pp. 50-52, 2006.

[23] L. Xi, Research on Safety Assessment Method of Urban Rail Transit Vehicles in Running Gear, Beijing Jiaotong University, 2011.

[24] L. Saihua, Identification Method and Its Application of Mountainous Freeway Accident Prone Section, Chang'an University, 2009.

[25] W. Qiang and W. Xianpu, "Study on speed limit control of expressway maintenance construction area," Traffic Information and Security, vol. 28, no. 1, pp. 124-129, 2010.

[26] Y. Ying, S. Yanting, and Y. Huazhi, "Risk evaluation and speed control of expressway entrance area," Journal of Traffic and Transportation Engineering, vol. no. 2, pp. 90-96, 2011.

[27] Z. Ming, Multi-Level Fuzzy Comprehensive Evaluation of Reservoir Induced Earthquake Risk Based on Association Rules And Cloud Model, Huazhong University of Science and Technology, 2013.

[28] Z. Zhengtao, Multi-Level Fuzzy Comprehensive Evaluation on Environmental Carrying Capacity of Island Tourism Based on Cloud Model, First Marine Research Institute, State Oceanic Administration, 2015. 


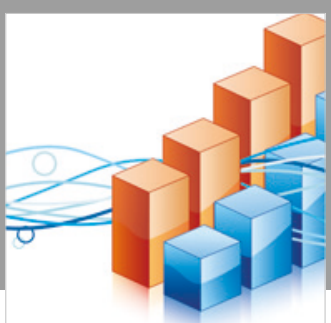

Advances in

Operations Research

\section{-n-m}
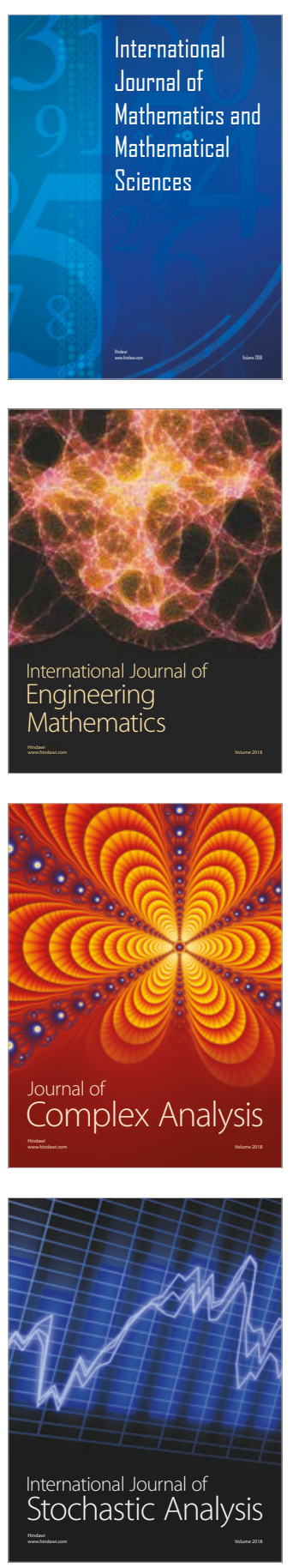
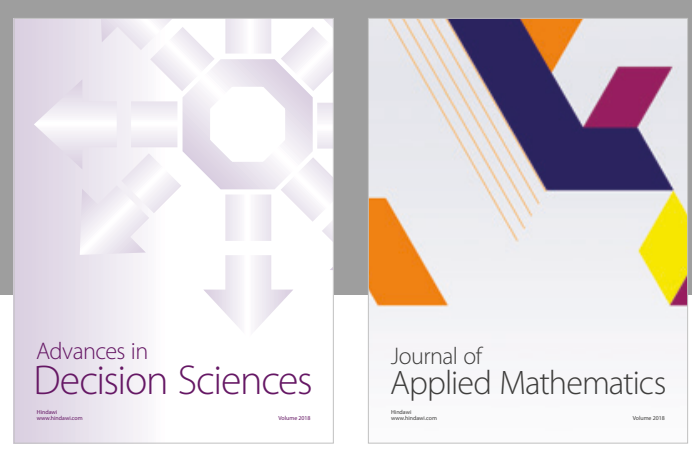

Journal of

Applied Mathematics
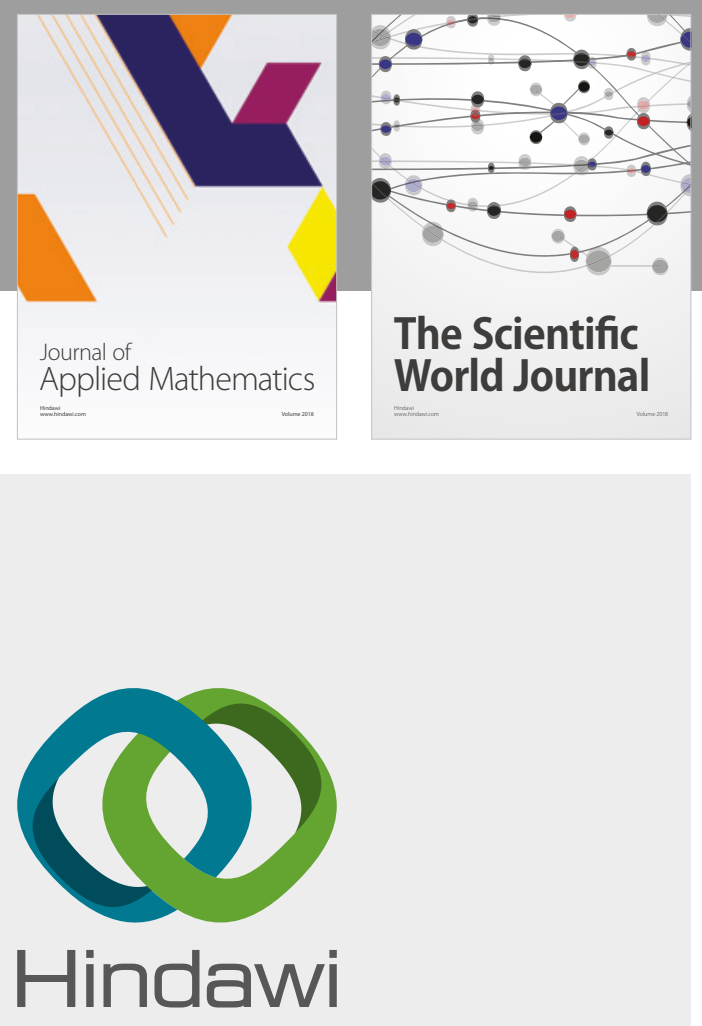

Submit your manuscripts at

www.hindawi.com

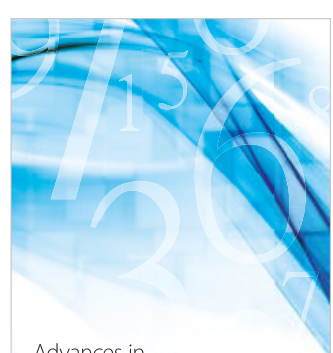

Advances in
Numerical Analysis
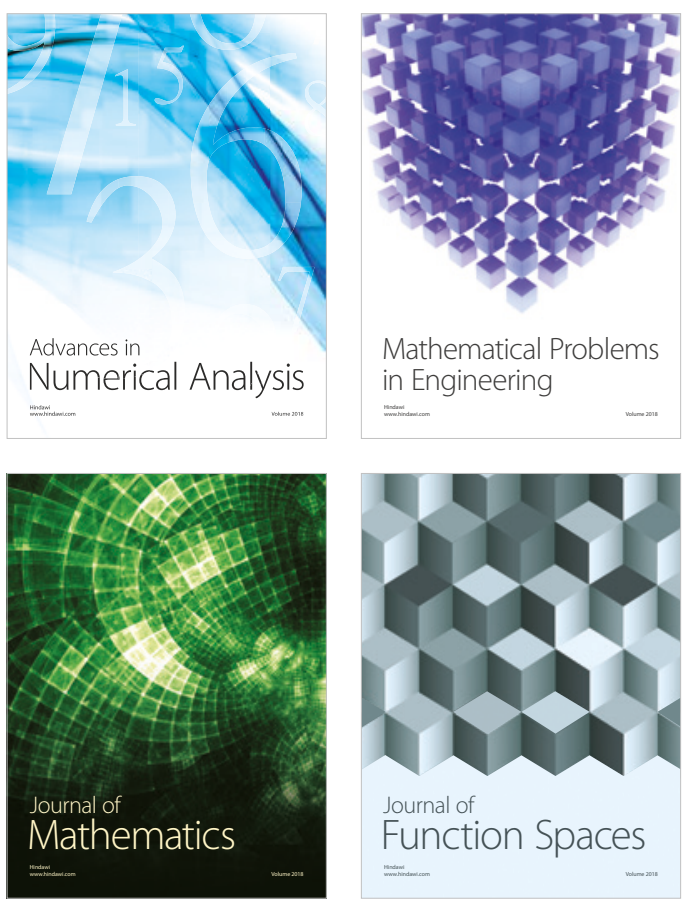

Mathematical Problems in Engineering

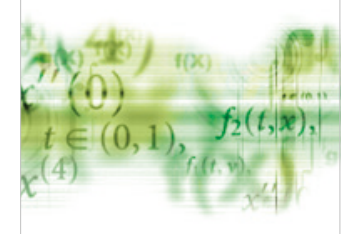

International Journal of

Differential Equations

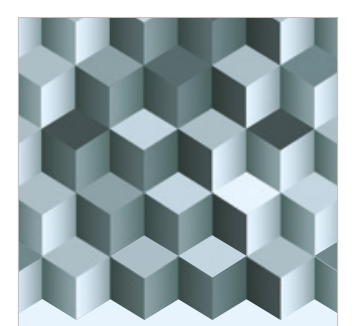

Journal of

Function Spaces

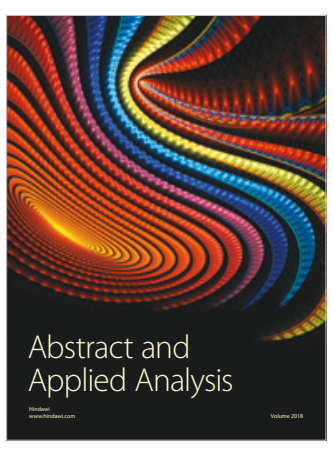

The Scientific

World Journal

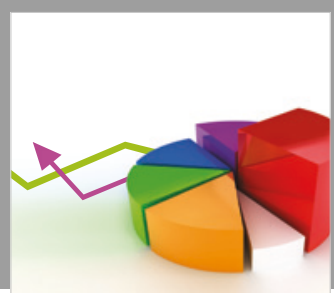

Journal of

Probability and Statistics
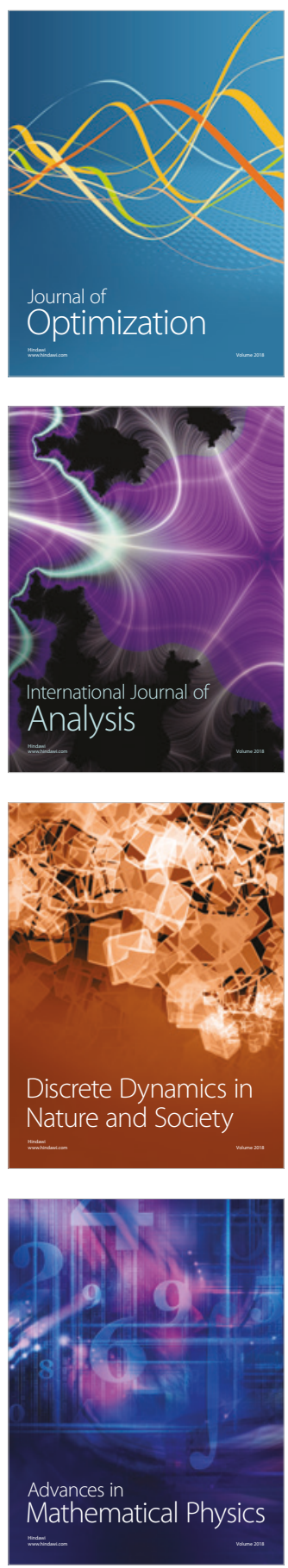